\title{
Improvements in Acne and Skin Oiliness with Tazarotene 0.045\% Lotion in Acne Patients with Oily Skin
}

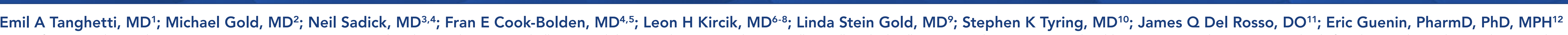

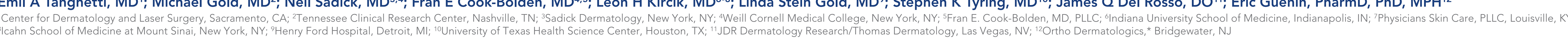

\section{SYNOPSIS}

- Excessive sebum production is a factor in facial acce development
frequent complaint of dermatology patients with or without acne

Larger pore sizes may be associated with higher rates of sebum production

- Skin oiliness and pore size can also differ by race, with larger amounts of sebum

- Topical retinoids are a mainstay of acne treatment, though they are associated with

- As vehicles formulated with emollients/moisturizers may reduce retinoid-associated
irritation,

- Though its effects on sebum production are unknown, the topical retinoid,
tazarotene $0.1 \%$ cream, has been shown to reduce apparent facial pore sire - The lower-dose $0.045 \%$ tazarotene polymeric lotion has also demonstrated efficacy
in reducing acre elesions and acne-induced sequalae such as hyperpigmentation
with good tolerability and safety profiles'

OBJECTIVE

- To evaluate efficacy, changes in skin oiliness, and safety/tolerability with tazarotene
$0.045 \%$ lotion in participants with acne and oily skin, including Black participants METHODS

- In two phase 3, double-blind, 12-week studies (NCT03168334; NCT03168321),
participants aged 29 years with moderate-to-severe acne were randomized 1:1 to ce-daily tazarotene $0.045 \%$ lotion or vehicle lotion

- Cerave hydrating cleanser and Cerave $\mathbb{E}^{\oplus}$ moisturizing lotion (L'Oreal, NY) were

- This pooled, post hoc analysis comprised participants categorized by self-reported
skin oiliness at baseline on the Acne-Specific Quality of Life questionnaire item 19 Scored from 0 (extremely oily) to 6 (not at all oily; Figure 1)

Only participants scoring 0-2 (oily skin) were included in this analysis

Coprimary endpoints were inflammatory/noninflammatory lesion counts and
treatment success $(22$-grade reduction from baseline in Evaluator's Global Severity

Score [EGSS] and a score of O [clearl or 1 [ [almost clear)

-Changes in skin oiliness, treatment-emergent adverse events (TEAEs), and

- A subset of participants with oily skin who self-reported race as Black were also
analyzed for changes in skin oiliness and cutaneous safety and tolerability IGURE 1. Oily Skin Assessment

19. In the past WEEK, how oily was your facial skin?

\section{RESULTS}

Demographics and Baseline Characteritics

had oily skin (45.6\%)

They had a mean age of 21.6 years, $73 \%$ were female, and $69 \%$ were White

Of 261 participants who identified as Black (ITT population), 150 had oily skin

They were slightly older (mean age 25.2 years), and a greater percentage were
female $82.7 \% \%$.

- Most had moderate EGSS at baseline (94.7\%)

Efficacy

In al particicants with oily skin, tazarotene $0.045 \%$ lotion provided significantly
greater least-squares mean percent reduction from baseline to week 12 in

icle $(P<0.001$, both; Figure 2$)$

- Treatment success rates at week 12 were significantly higher for all tazarotene
treated participants vs vehicle $(29.8 \%$ vs $19.2 \% ; P<0.01)$

These results are similar to those in the overall phase 3 pooled population for
lesion reductions (see figure 2 footnote) and treatment success rates at week 12

FIGURE 2. Reductions in Acne Lesion Counts by Visit in All
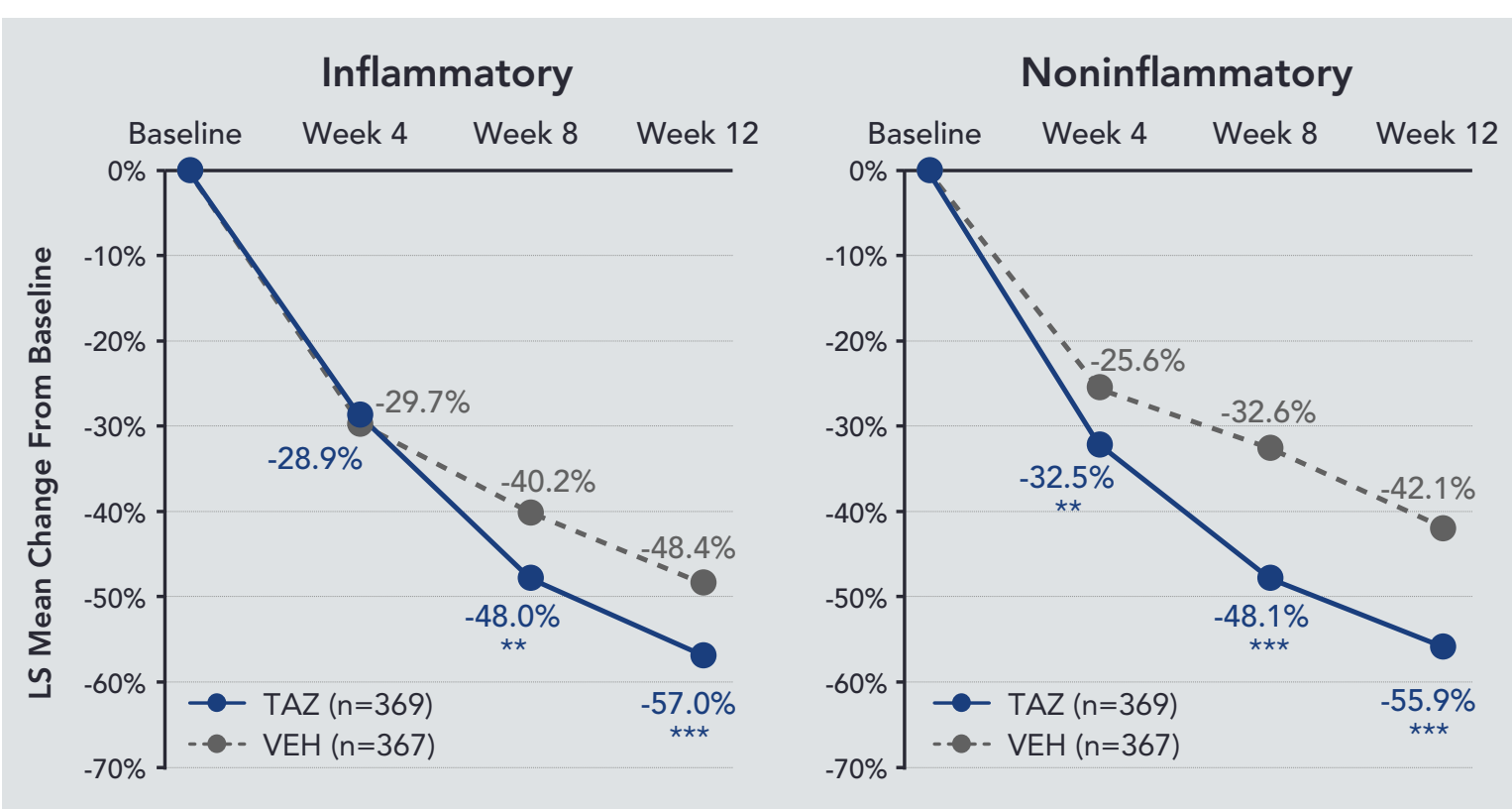

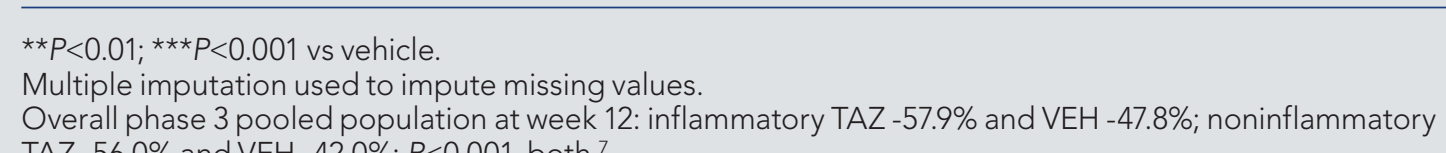

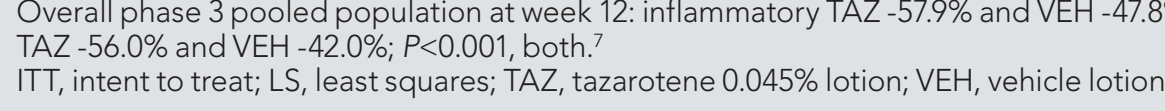

Skin Oiliness

- Most participants reported an improvement in skin oiliness to "moderately" or
"low/not" oily with tazarotene $0.045 \%$ and vehicle; numerically more participants reported an improvement to "low/not" oily skin with tazarotene than vehicle (Figure 3) - Most Black participants with oily skin also reported an improvement in skin oiliness
to "moderately" or "low/not" oily with tazarotene and vehicle, with a numerically
FIGURE 3. Improvements in Oily Skin from Baseline to Week 12

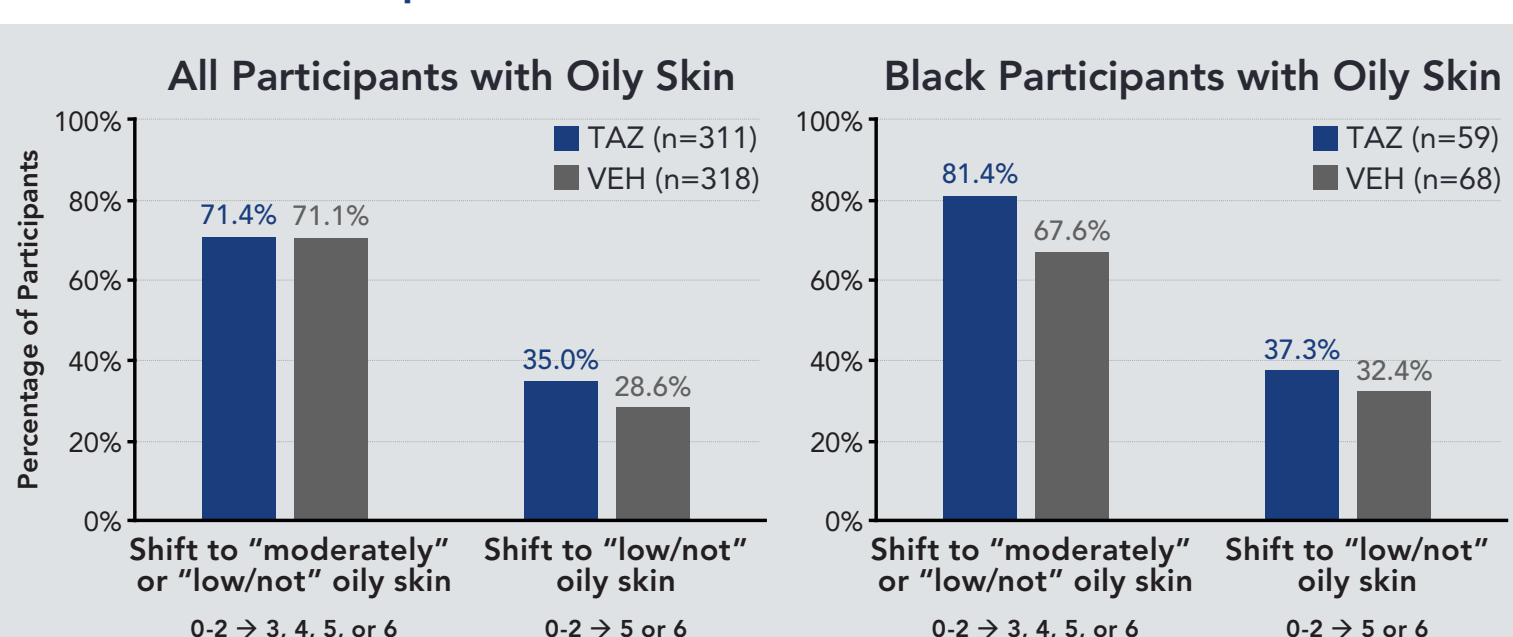

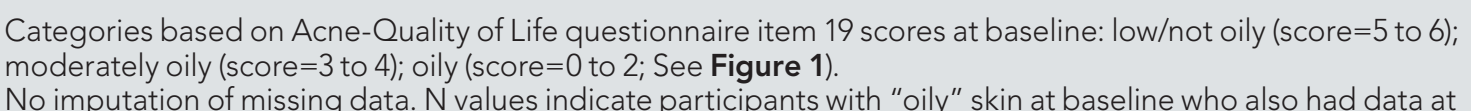

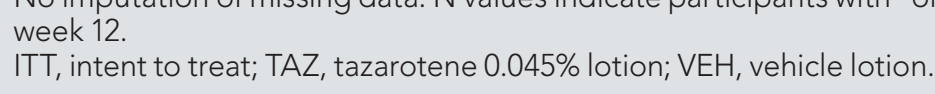

Safety and Tolerability

- TEAE rates with tazarotene in all oily-skin patients $(n=359 ;$ s safety population) were
similar to those observed in the overall tazarotetene-treated population $(n=779$; safety

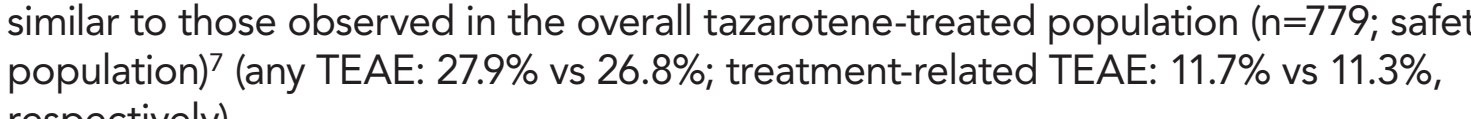

- The percentage of tazarotene-treated oily skin participants reporting "none" on cutaneous safety and tolerability assessments at
baseline values for most assessments (Figure 4)

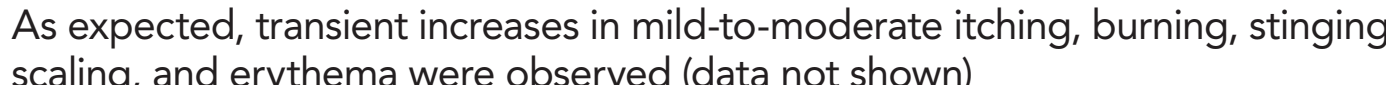

,

- Similar trends were observed in Black participants with oily skin (tazaratonene $n=70$,
vehicle $n=75$; safety population), though Black participants had greater improvements sin hyperpigmentation and fewer reports of erythema and scaling at
week 12 (Figure 4 )

FIGURE 4. Cutaneous Safety and Tolerability
(Safety Population)

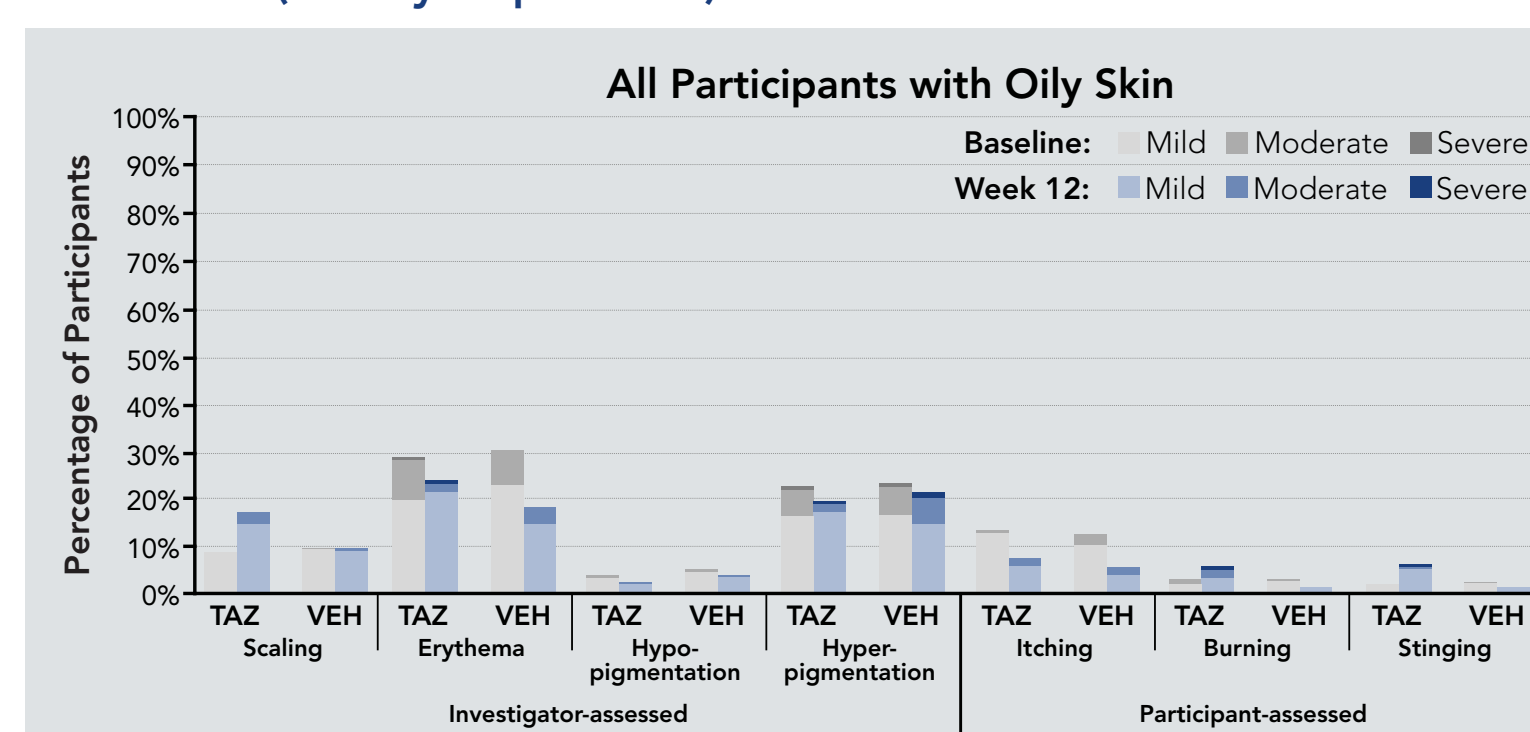

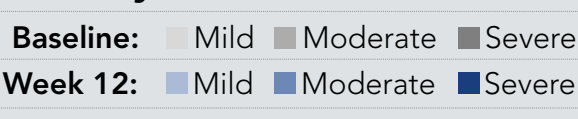

CONCLUSIONS

- Nearly three-fourths of all participants treated with tazarotene $0.045 \%$ lotion-including Black participants-had subjective reductions in skin oiliness by week 12 , with over a third reporting low/not oily skin

- It is possible the improvements in oiliness observed with tazarotene lotion were due to the unique, non-greasy excipients/emulsifiers contained in the polymeric emulsion lotion vehicle this emulsion technology provides fast, uniform, and complete release of the humectants, oil droplets, and other excipients contained in the vehicle onto the skin"

- Tazarotene lotion also demonstrated efficacy and safety in the treatment of moderate-tosevere acne in participants with oily skin, with rates similar to the overall population

- Once-daily treatment with tazarotene $0.045 \%$ lotion may help improve patient-perceived skin oiliness in those with moderate-to-severe acne

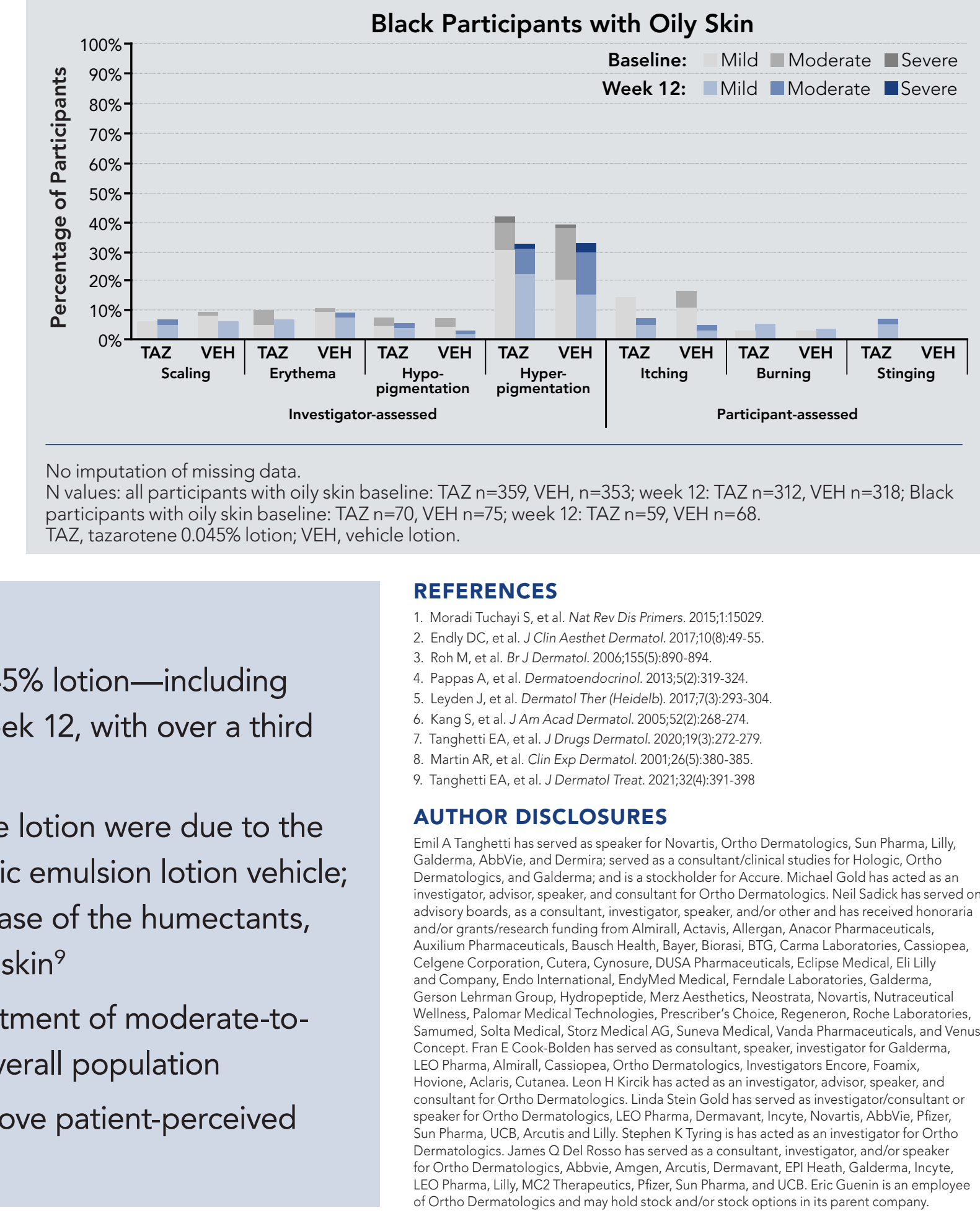

